
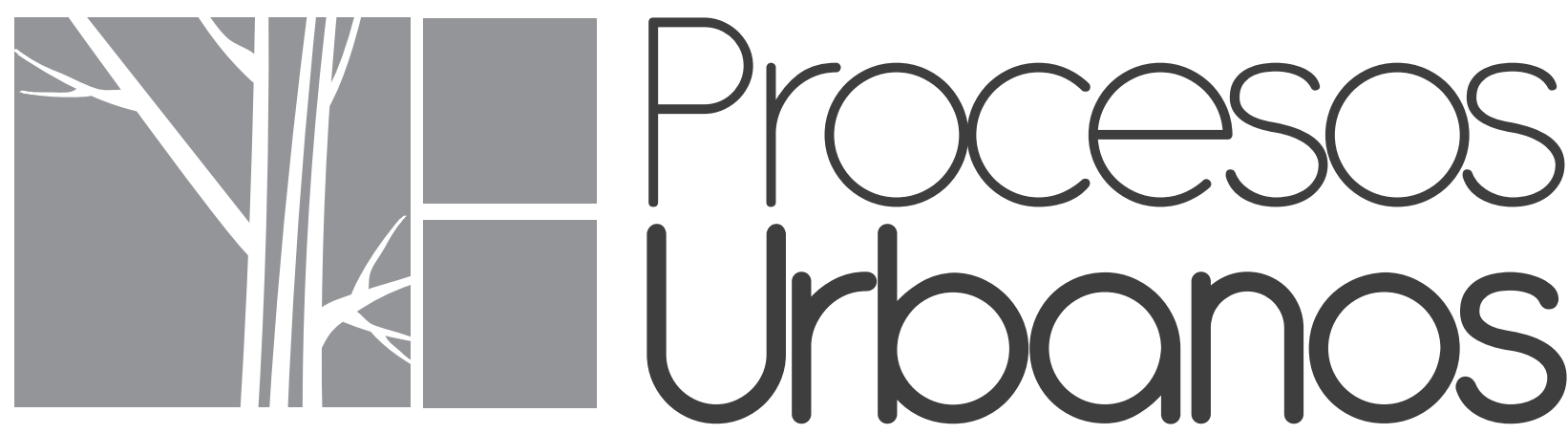

Revista de Divulgación Científica 


\section{Revista Procesos Urbanos}

Facultad de Ciencias Básicas, Ingeniería y Arquitectura.

ISSN: 2422-085X

Sincelejo, Enero - Diciembre de 2014

Piedad Martínez Carazo

Rectora

\section{Lidia Flórez de Albis}

Vicerrectora Académica

\section{Jhon Víctor Vidal}

Director de Investigaciones

\section{Pedro Arturo Martínez Osorio}

Editor

\section{Comité Editorial:}

PhD. Alexander Niño Soto. Universidad Antonio Nariño

PhD. Jorge Ramírez Nieto. Universidad Nacional de Colombia

Msc. Juan Carlos Pergolis. Universidad Católica de Colombia

PhD. Piedad Martínez Carazo. Corporación Universitaria del Caribe, CECAR

PhD. Emiro F. Martínez. Universidad de York, Canadá

\section{Comité Científico:}

Msc. Gloria Aponte García. UPB, Medellín

Msc. Jorge Vásquez Muñoz. UPB, Medellín

PhD. Jorge Gómez Ricardo, Universidad del Magdalena

\section{Editorial CECAR}

\section{Libia Narváez Barbosa}

Coordinadora Editorial

\section{Eduardo Támara Galván}

Corrector de Estilo

\section{Alejandrina Jaramillo y Roger Goez Castillo}

Diseño Gráfico

Diagramación e Impresión:GRÁFICAS DEL CARIBE S.A.S.

Cra. 1B No. 40-42 Montería Tel. (57) (4) 7826622 Telefax (57) (4) 7817112

Email: diseno@graficaribe.co

\section{Dirección:}

Carretera Troncal de Occidente

Kilómetro 1 Vía a Corozal

Tel: 280402928040172804018 Ext 1115

www.cecar.edu.co

http://cecar.edu.co/revista-procesos-urbanos 


\title{
BUENOS EJEMPLOS EN LA PRÁCTICA DE LA CONSTRUCCIÓN SOSTENIBLE EN EL MUNDO CONTEMPORÁNEO ${ }^{1}$
}

\author{
Good examples in practice of sustainable construction in the contemporary world \\ Josué Ramón Cárdenas Priego²
}

Fecha: Recibido enero 31 de 2014 / Aceptado abril 26 de 2014

\section{RESUMEN}

En la última década la industria de la vivienda en América Latina ha logrado reducir el rezago habitacional considerablemente, pero en el camino por abatir la falta de viviendas se ha generado un crecimiento urbano desordenado que está deteriorando la calidad de vida de la población. En este contexto ¿es posible hablar de desarrollo sustentable?. El objetivo del presente artículo es presentar un recorrido por el estado del arte en materia del desarrollo de arquitecturas, enfocadas bajo el concepto de desarrollo sostenible a nivel mundial. Nace de una investigación de corte cualitativo, en la que se utilizó una revisión documental sobre el concepto de desarrollo sostenible y las buenas prácticas de éste en la arquitectura y el urbanismo de hoy; en ella la observación directa se convierte en la herramienta precisa para constatar las potencialidades de los ejemplos que se ofrecen como representativos de unas buenas prácticas de una arquitectura sostenible en el mundo contemporáneo. La discusión que plantea, gira en torno a la desmitificación de algunas interpretaciones forjadas a partir del concepto de desarrollo sostenible, lo que genera una crítica a la forma como ha evolucionado el concepto, y se presentan algunos buenos ejemplos de cómo desarrollar una arquitectura amigable con el planeta. Como conclusión se plantea llamar al urbanismo sin ningún adherente de (bioclimático, ecológico, sustentable), un urbanismo capaz de responder a las condiciones singulares del clima y territorio, entendiendo que cada situación geográfica genera un diseño característico y diferente con respecto a otros lugares.

Palabras Clave: Urbanismo, sostenibilidad, medio ambiente, energía alternativa, eficiencia energética.

\section{ABSTRACT}

In the last decade the industry of the housing in Latin America has managed to reduce and leave behind housing deficit considerably, but in the way for bringing down the lack of housings there has been

\footnotetext{
${ }^{1}$ El presente artículo nace de una investigación de corte cualitativo, utilizando una revisión documental sobre el concepto de desarrollo sostenible y las buenas prácticas de éste en la Arquitectura y el Urbanismo donde la observación directa se convierte en la herramienta precisa para constatar las potencialidades de los ejemplos que se ofrecen como representativos de unas buenas prácticas de una arquitectura sostenible en el mundo contemporáneo. ${ }^{2}$ Licenciado en Arquitectura de la Universidad Autónoma de Tamaulipas de la Unidad Académica de Arquitectura, Diseño y Urbanismo en México. 2005. Especialista en Bioconstrucción y Energía edificatoria de la Escuela Técnica Superior de Arquitectura de Madrid - ETSAM. Universidad Politécnica de Madrid, 2006 Magíster en Medio Ambiente y Arquitectura Bioclimática de la Escuela Técnica Superior de Arquitectura de Madrid - ETSAM. Universidad Politécnica de Madrid, 2007. Docente catedrático y sinodal de la licenciatura en Arquitectura, Instituto de Estudios Superiores de Tamaulipas - IEST, Tampico México. Email: cardenas@arbico.com.mx
} 
generated an disorder urban growth that is spoiling the quality of life of the population, in this context is it possible to speak about sustainable development? The aim of the present article is to present a tour for the state of the art as for the development of architectures, focused under the concept of sustainable development worldwide. It begins of an investigation of qualitative cut, in which a documentary review was in use on the concept of sustainable development and the good practices of this one in the architecture and the today urbanism, in it the direct observation turns into the precise tool to state the potentials of the examples that offer like representative as a few good practices of a sustainable architecture in the contemporary world. The discussion that it raises, turns environment to the debunking of some interpretations developed from the concept of sustainable development, which generates a critique to the form since has evolved the concept, and they present some good examples of how developing an friendly architecture with the planet. As a conclusion considers to call to the urbanism without any adherent of (bioclimatic, ecological, sustainable), an urbanism capable of answering to the singular conditions of the climate and territory, understanding that every geographical situation generates a typical and different design with regard to other places.

Keywords: Urbanism, sustainability, environment, alternative energy, energy efficiency.

\section{INTRODUCCIÓN}

En la última década la industria de la vivienda en América Latina ha logrado reducir el rezago habitacional considerablemente, pero en el camino por abatir la falta de viviendas se ha generado un crecimiento urbano desordenado que está deteriorando la calidad de vida de la población. Los países latinoamericanos que eran eminentemente rurales, pasaron a ser naciones con prevalencia urbana sin la planeación que esto requiere. Según estudios realizados por la Organización de las Naciones Unidas
- ONU, en el 2005 el 80\% de la población ya habitaba en zonas metropolitanas, como la ciudad de México, con una población mayor a 21 millones, de habitantes, Sao Paulo con 18 millones o Buenos Aires con 12 millones pasaron de ser metrópolis a una megalópolis (Annan. K. 2005, p.125).

En estudios realizados por la Organización Mundial para las Migraciones - OIM, el crecimiento desmedido de la población está muy ligado con problemas de pobreza y violencia; cerca del $40 \%$ de los residentes urbanos de América latina y el Caribe viven hoy en asentamientos marginales, espontáneos o ilegales, lo que llamamos tugurio, favelas o ciudades dormitorios (Wolfensohn. J. 2005). La mayoría de estos asentamientos marginales están localizados en áreas en las que los problemas ambientales son identificables, como la contaminación del suelo, del aire, del agua, con procesos de erosión debido a la quema y tala de bosques que conllevan a riesgos de deslaves, inundaciones, problemas de salud y en muchas ocasiones, con un costo de vidas humanas.

Teniendo como base el problema de la visión del siglo $X X$, en el cual la zonificación se impone ante la ciudad tradicional de usos múltiples, sectorizando las áreas residenciales, comerciales e industriales, es posible ver que se ha generado que la ciudad esté funcionando por horas en las diferentes zonas, lo cual es absurdo y obliga a la población a movilizarse a los sitios de trabajo y de ocio, lo que genera un excesivo parque vehicular causante de congestionamiento, accidentalidad, consumo de combustibles fósiles, contaminación. En ciudades como Cali, en horas de congestión vehicular, la velocidad promedio no alcanza los $12 \mathrm{~km} / \mathrm{h}$ (Leal.G.2009, P. 18).

Otro problema muy preocupante es que el $20 \%$ de la población no cuenta con agua potable (se dice que las guerras del siglo XXI serán provocadas por la escasez de agua) sumado a una prestación de servicios básicos de infraestructura urbana que sigue siendo precaria, ya que 3 de cada 5 personas no cuentan con servicio de alcantarillado. 
El tratamiento de los desechos sólidos representa un problema; hoy en día la generación per- cápita de residuos varía entre el 0.5 a $1 \mathrm{~kg}$ diarios; el problema no es solo la cantidad, sino en la composición de los desechos que cada vez son menos biodegradables, esto sumado a un mal manejo de la basura y a la ineficacia gubernamental para establecer programas y leyes que hagan responsables a todos sobre esta situación. Los grandes volúmenes de desechos provocan que los sitios de acopio sean ya insuficientes y se acumulen las basuras en avenidas, parques y en casi todo el espacio urbano, con la consecuencia de afearla y no hacerla atractiva para su inversión o como destino turístico.

Todos estos problemas los podemos enmarcar en las alteraciones que causan la mala planificación, dirección y diseño de una ciudad, de cuyos resultados son establecer los síntomas de una patología urbana en la que nuestras ciudades actúan como ecosistemas lineales cuya secuencia sería extraer, producir, vender, consumir y desechar.

Extraemos $\longrightarrow$ Producimos $\longrightarrow$ Vendemos $\longrightarrow$ Consumimos $\longrightarrow$ Desechamos

Todo esto tiende a empeorar si no empezamos ya a enfrentar los problemas que hoy padecemos. Ante esta problemática, es posible plantear un gran interrogante de fondo ¿Se puede hablar de desarrollo sustentable inmerso en una grave crisis como la actual?

\section{METODOLOGÍA}

El presente artículo nace de una investigación de corte cualitativo, en la que se utilizó una revisión documental sobre el concepto de desarrollo sostenible y las buenas prácticas de éste en la Arquitectura y el Urbanismo. En ella la observación directa se convierte en la herramienta precisa para constatar las potencialidades de los ejemplos que se ofrecen como representativos de unas buenas prácticas de una arquitectura sostenible en el mundo contemporáneo.

\section{RESULTADOS}

Antelagravecrisisdelmundocontemporáneo, es posible hablar de desarrollo sustentable; es un imperativo, porque la situación en que nos encontramos es apenas un anticipo de otra peor, en avanzado estado de gestación. La sociedad "global" se está enfrentando a problemas económicos y financieros (sin nombrar el inmobiliario en algunas zonas), dificultades que están ocultando la más grave crisis todavía por llegar: la ecológica.

Haciendo un recuento de lo que se ha hecho a nivel global en materia de sustentabilidad, se aprecia que desde hace más de tres décadas en los años 70, la protección del medio ambiente surgió de las campañas y agendas políticas de distintos países. Para 1983 la Organización de las Naciones Unidas reunida en Estocolmo, había establecido la comisión mundial para el medio ambiente y desarrollo sustentable.

En 1987 la ONU emitió un documento llamado Nuestro Futuro Común, mejor conocido como Informe BRUDTLAND, que fue encabezado por la dra. Harlem Brudtland quien utilizó por primera vez el término desarrollo sustentable, definido como aquel que satisface las necesidades del presente sin comprometer las necesidades de las futuras generaciones (Brudtland. H. 1987, P. 56).

Es cuando nace el esquema con los tres pilares de desarrollo sostenible interrelacionando los aspectos social, económico y ambiental. Este concepto fue el comienzo para iniciar a descifrar un tema tan complejo, pero hacía falta una herramienta de medición. En 1996, los investigadores Mathis Wackernagel y William Rees definieron el concepto Huella Ecológica como: "el área de territorio productivo o ecosistema acuático necesario para producir los recursos utilizados y para asimilar los residuos producidos por una población definida, con un nivel de vida específico, donde sea que se encuentre esta área" (Wackernagel. M. - Rees. W. 1996, P. 86).

LA HUELLA ECOLÓGICA $=$ SUELO,
SUMINISTROS URBANOS + SUELO
DESECHOS URBANOS. 
En otros términos es un instrumento que mide, el peso de nuestro modo de vida o sea de nuestra manera de producir y consumir sobre el planeta. Claro que el planeta tiene sus límites representa 51,000 millones de hectáreas, sin embargo, se ha calculado que el espacio bioproductivo disponible es de 12.000 millones de hectáreas, y como somos 6.500 millones de personas nos queda de 1.8 hectáreas de espacio bioproductivo disponible por persona (Wolfensohn, J. 2005. P. 220).

Por si fuera poco, esta excesiva explotación del medio ambiente no se lleva a cabo de manera uniforme en la totalidad del planeta: por poner un ejemplo, Estados Unidos de Norteamérica utiliza el $120 \%$ de su capacidad, mientras que Perú solo usa el 14\%, o Gamboa el 6\% (Linares. J. 2010, P. 285). Existen por lo tanto dos problemas diferentes, pero perfectamente interrelacionados: el primero se refiere a que hemos sobrepasado la capacidad de carga del planeta. Pero el segundo, cada vez más evidente, es que esta explotación excesiva es de unos pocos a costa de otros.

En el año 2012 se llevó acabo la reunión de las Naciones Unidas sobre el desarrollo sustentable, hecho que marcó el vigésimo aniversario de la primera cumbre de la tierra donde se trataron temas de suma importancia como las agendas 21 locales y los compromisos adquiridos.

\section{¿Qué se entiende por sostenibilidad?}

Hoy en día el uso excesivo de la palabra desarrollo sustentable ha llevado a vaciarla de contenido, quitándole su esencia, sus valores y equivocando el propósito por el cual fue creada. Hablar de sostenibilidad, cada vez tiene menos sentido porque el término se lo han apropiado las instituciones que más contribuyen a la insostenibilidad.

Hoy cualquier cosa es "sostenible"; desde un automóvil que no presenta ninguna diferencia con otro "insostenible". Cierto elitista hotel en algún rincón hasta entonces virgen del planeta, que se limita a los aspectos más estéticos, entendidos de la forma más banal, como pura apariencia.
Un ecologismo "cosmético" que no pasa, las más de las veces, de "esconder el polvo debajo de la alfombra", o lo que es lo mismo, exportar los residuos, mientras sigue deglutiendo bulímicamente recursos naturales del lugar.

Es una fórmula que vende imagen, que añade valor a cualquier producto; pero nada más. Una falsificación peor, un urbanismo tecnológico, que ha hecho de la eficiencia, bandera. No se trata de cambiar nada, sino de mejorar técnicamente nuestro mundo: la tecnología tiene la llave, la varita mágica.

¿Qué agotamos los combustibles fósiles en un absurdo derroche? "No problem": la fusión nuclear, la recreación del sol en la tierra, nos permitirá disponer ilimitadamente de energía, y quizás como paso previo, las pilas de hidrógeno o los biocombustibles suplirán la transición, aunque ello suponga condenar al hambre a millones de personas.

En México tenemos 150 proyectos postulados para recibir la certificación LEED, mientras que hace dos años sólo hubo 20 proyectos que aspiraban al reconocimiento. Esto nos puede dar una buena idea de que el negocio verde es provechoso.

\section{¿Pero que es LEED?}

LEED es una poderosa herramienta creada en 1998 por el Consejo de la construcción verde de los Estados Unidos, la certificación que implementa este sistema evalúa seis aspectos de los edificios:

1. Sostenibilidad de la parcela.

2. La eficiencia en agua.

3. La eficiencia energética y el uso de renovables.

4. Los materiales y recursos.

5. La calidad medioambiental interior.

6. La innovación en tecnología y procesos.

La cadena City Express tiene 11 de sus 59 hoteles en México en proceso de certificación LEED. En 2012, el proyecto se extenderá a dos unidades en Costa Rica, una en Chile y otra en Colombia. City San Luis Potosí fue el primer inmueble que obtuvo el reconocimiento internacional (2010) y City 
Express Reforma El Ángel, el primero que generó su propia energía, por medio de micro turbinas que funcionan con gas LP. City Express utiliza productos reciclados, como el triplay de los muebles, el PVC de la cancelería o las ocho botellas de polietileno tereftalato (PET) que hay por cada metro cuadrado de tapiz.

De nuevo LEED es una herramienta que nos ayuda a fijar parámetros para una construcción sostenible, pero sin un sentido crítico y un cambio de paradigma del arquitecto, seguiremos construyendo edificios con un coste elevado de construcción, añadiendo nuevas tecnologías en lugar de buscar lo más básico con un diseño pasivo.

A continuación traigo unos ejemplos que en mis viajes considero que han logrado con su diseño básico y pasivo una verdadera sostenibilidad desde la arquitectura.

\section{Primer ejemplo (Ecobarrio de Kronsverg)}

Está ubicado al sureste de Hannover en la ciudad de Kronsverg; fue construido según los últimos conocimientos sobre edificación ecológica y habitar en el sentido de las agendas 21 europeas, siendo los requisitos de la planeación urbana una construcción que ahorre superficie, tráfico no contaminante, calidad en espacios libres así como una vecindad para vivir y trabajar.

Para la realización de la construcción se contrataron a más de 40 inversores, siendo los proyectos detallados por el ayuntamiento, el cual fue asesorado por un concejo consultivo de planificación local.

La infraestructura se originó simultáneamente con la construcción de las viviendas. Las instalaciones públicas fueron financiadas por la venta de terrenos de la ciudad a inversionistas privados.

La idea principal del desarrollo urbano sostenible son construcciones compactas y de alta densidad de edificación con arquitectura variada y calidad de habitar. Fueron edificadas solo casas unifamiliares las cuales forman aproximadamente 10\% de todas las viviendas existentes, y el $90 \%$ son edificios multifamiliares.

Se realizaron viviendas que facilitaran a las personas minusválidas el poder vivir independientemente así como de considerar las costumbres de culturas diferentes, para promover la convivencia de familias alemanas e inmigrantes.

Alrededor de una plaza pública se agrupan los siguientes centros: centro comercial, centro evangelista eclesiástico, centro sanitario, tiendas, cafeterías, restaurantes, entre otras zonas para el ocio y el disfrute urbano.

Krokus (Centro Socio cultural del barrio) es un lugar de encuentro y al mismo tiempo un fórum central para el trabajo comunitario el cual combina tareas sociales con temas culturales y ecológicos.

Enseguida describiré varios aspectos que, basado en mi experiencia, marcaron la diferencia desde su planificación, diseño y construcción.

\section{Planificación de la circulación}

El flujo principal del tráfico se canaliza a lo largo del borde de la zona junto a la línea de tranvía; el planteamiento de calles estrechas no deja que se forme tráfico; se han dispuesto carriles bici que atraviesan el distrito de norte a sur; las plazas de estacionamiento se encuentran en pequeños aparcamientos subterráneos aprovechando, la topografía.

\section{Optimización ecológica}

La integración de aerogeneradores con potencias de $1.8 \mathrm{~mW}$.

La instalación de colectores solares con más de 1,350 m2 integrados a los tejados.

Central de calefacción.

Articulación con los inversionistas privados, los cuales se comprometen a construir casas que solo consuman $15 \mathrm{~kW} / \mathrm{m} 2$ al año. 
PROCESOS URBANOS - Revista de Divulgación Científica Vol. 1 Enero - Diciembre 2014 (113 - 120)

\section{Gestión del agua}

En Kronsverg se pudo conservar ampliamente al balance natural del agua.

En espacios de zonas públicas se recoge el agua de lluvia en el sistema Mulden Rigolen siendo el agua utilizada como elemento de diseño urbano en forma de estanques o corriente de agua.

\section{Gestión de los residuos}

Cerca de las viviendas se instalaron contenedores con un diseño que facilita el depositar el residuo una vez clasificado en orgánico, papel, vidrio. Este sistema garantiza una amplia clasificación de los residuos. Así también un programa de fomento que apoyaba el compostaje propio de jardines.

Bajo el lema "arréglalo, no lo tires" se ha creado una red de servicios de reparación y modificación.

\section{Gestión de la tierra}

La tierra excavada fue empleada para la creación de biotopos, para la modelación de dos colinas miradores, asi también como para la construcción de terraplenes anti ruido a lo largo de la autopista, logrando con esto evitar aproximadamente 100.000 viajes de camiones por zonas pobladas.

\section{Segundo ejemplo (Ecobulevar de Vallecas)}

La propuesta tiene por objeto el acondicionamiento bioclimático del Bulevar principal del Ensanche de Vallecas, un espacio exterior de $550 \times 50 \times 17 \mathrm{~m}(467.500 \mathrm{~m} 3)$.

Se trata de una experiencia innovadora de diseño urbano que pretende mejorar el confort ambiental, promover el intercambio social y ser más sostenible que los modelos convencionales de crecimiento de la ciudad.

Los criterios medioambientales y de sostenibilidad subyacen en todas las determinaciones del proyecto: los materiales empleados (en su mayoría de origen reciclado), la utilización de energías alternativas, la climatización por sistemas pasivos, la optimización de los recursos, el carácter social o la nueva disposición asimétrica de las vías de tráfico rodado.

Proceso de gestión (reciclando la no-ciudad)

Se convoca el concurso de ideas del Ecobulevar con un doble objetivo: uno de carácter social que persigue la generación de actividad y otro de carácter ambiental, el acondicionamiento climático de un espacio exterior.

El espacio público es un espacio de todos, que debe funcionar como soporte para múltiples actividades y acontecimientos, más allá de todo aquello planificable; un espacio donde el ciudadano pueda actuar en libertad y en el que tenga cabida la espontaneidad. La propuesta trata de compensar la falta de actividad, y surge a partir del interés por solucionar el problema desde el primer momento, siendo conscientes de que el mejor acondicionamiento para un espacio público sería un arbolado suficientemente denso y de buen porte, material con el que no se puede contar hasta dentro de unos quince o veinte años. Era pues necesaria una actuación "de urgencia", que fuera capaz de funcionar como un bosque lo haría en el futuro.

Se opta por una estrategia de concentración, que interviene y acondiciona áreas concretas, dotándolas de un mayor confort climático, y sirven así como germen de un proceso regenerador del espacio público. No pensamos que sea necesario un edificio, sino un lugar para los ciudadanos, cuya forma venga definida por la propia actividad de cada momento.

Tres pabellones o árboles de aire funcionan como soportes abiertos a múltiples actividades elegidas por los usuarios. Instalados en la no-ciudad como prótesis temporales, se usarán sólo hasta que la tara de inactividad y de acondicionamiento climático, se haya corregido. Transcurrido el tiempo suficiente, estos dispositivos deberían desmontarse, permaneciendo los antiguos recintos como claros en el bosque. 
El árbol de aire es una estructura ligera, desmontable y autosuficiente energéticamente, que sólo consume lo que es capaz de producir mediante sistemas de captación de energía solar fotovoltaica. La venta de esta energía a la red eléctrica genera un superávit en el balance anual, que se reinvierte en el mantenimiento de la propia infraestructura. Esto último no es otra cosa que un modelo de gestión de los recursos de un proyecto en el tiempo.

El uso de la tecnología juega en este proyecto un papel crítico y decisivo adecuándose a un contexto real y concreto. El potencial arquitectónico de la tecnología reside en su reprogramación y combinación con otros elementos configurando verdaderos readymades arquitectónicos. En este caso se toman prestadas técnicas de climatización usadas habitualmente por la industria agrícola.

La autonomía con la que funcionan los árboles de aire los convierte en objetos de carácter exportable, siendo posible su reinstalación en lugares similares o en otro tipo de situaciones que necesiten un proceso de regeneración de la actividad urbana (nuevos ensanches, parques degradados, plazas).

El objetivo en el proyecto de Vallecas es crear una atmósfera que invite y haga propicia la actividad en un espacio público urbano. Los sencillos sistemas de climatización instalados en los árboles de aire, son de tipo evapotranspirativos, aplicados frecuentemente en invernaderos. Esta práctica aerotécnica o acondicionamiento artificial, no forma parte de una estrategia comercial, antes bien, trata de desligar el binomio ocio-consumo, y reactivar el espacio público, creando entornos acondicionados (8$10^{\circ} \mathrm{C}$ más fresco que en el resto de la calle, en verano), en los que el ciudadano forme de nuevo parte activa del espacio público.

Plantearon una serie de intervenciones superficiales (asociadas a la ejecución de los árboles de aire) que constituyen un ejercicio de reciclaje y reprogramación de la ciudad, conceptualmente extrapolable a cualquier intervención urbana en espacios públicos de la ciudad consolidada. Creemos posible la regeneración de espacios urbanos utilizando técnicas no invasivas, sin generar residuos ni consumir ingentes cantidades de energía y presupuesto. El arquitecto contemporáneo debe ser un gestor que optimice los recursos y establezca las prioridades encaminadas a conseguir lo más con menos, un efecto máximo con una intervención mínima, que tiene mucho de sostenible y poco de minimalista.

Entonces, ¿Cuál es la metodología que debemos seguir? Para comenzar, llamar al urbanismo sin ningún adherente de (bioclimático, ecológico, sustentable) simplemente. URBANISMO.

Este urbanismo debe responder a las condiciones singulares del clima y territorio, entendiendo que cada situación geográfica genera un diseño característico y diferente con respecto a otros lugares.

\section{CONCLUSIONES}

1. Este urbanismo se enmarca dentro de la planificación del desarrollo sostenible, cuyo objetivo es el de mejorar la calidad de vida de las personas aprovechando al máximo los recursos disponibles y controlando los efectos perniciosos sobre el medio ambiente en todas las escalas, (recursos del soporte, del clima, energéticos, paisajísticos e incluso, socio-económicos).

2. Los principios generadores del urbanismo bioclimático se pueden resumir en los siguientes puntos:

a) Un trazado viario estructurante que responda a criterios de soleamiento $y$ viento.

b) Calles adaptadas a la topografía, buscando las orientaciones óptimas de soleamiento y viento.

c) Zonas verdes adecuadas a las necesidades de humedad y evaporación ambiental (en superficie, conexión y especies vegetales apropiadas). 
PROCESOS URBANOS - Revista de Divulgación Científica Vol. 1 Enero - Diciembre 2014 (113 - 120)

d) Morfología urbana que genere fachadas bien orientadas.

e) Tipología edificatoria diversa y adecuada a las condiciones de sol y viento del lugar.

f) Compactación de la ciudad con base en pocas acciones concentradas.

g) Promover los usos múltiples.

3. Para lograr esto se desarrolla y se propone la siguiente metodología:

a) Establecer una síntesis de los condicionantes del medio, de planos o textos sintéticos de diagnosis.

b) Establecer las determinaciones que condicionan el microclima local, sobre todo las de viento y sol, al objeto de cuantificar las necesidades locales y, así, poder formular las principales estrategias para conseguir los objetivos que se persiguen.

c) Por último, estas estrategias generales se plasmarán en los documentos de planificación territorial o urbana que sean oportunos, articulados mediante los sistemas generales urbanos (red viaria, equipamiento y red de zonas verdes y espacios libres), y la redacción de ordenanzas ambientales principalmente.

\section{REFERENCIAS}

Annan, Koffi, A (2005) Objetivos del desarrollo del milenio, informe del 2005. Editorial Banco Mundial.

Higuera. Ester. Urbanismo Bioclimático. Editorial Gustavo Gili. 2006.

Leal del Castillo, Gabriel (2006). Ciudad, Medio Ambiente y Sostenibilidad. Ecoe ediciones.

Organización de las Naciones Unidas. Guías de la Agenda 21. Editorial CNUMAD, 1992.

Wackernagel, Mathis y William Rees (2001). Nuestra Huella Ecológica, Reduciendo el Impacto Humano sobre la Tierra. Ediciones LOM. Santiago de Chile, 2001.

Wolfensohn, James D. (2005) Informe sobre el desarrollo Mundial. Editorial Banco Mundial. 